\title{
A Novel Method of Preventing Pharyngocutaneous Fistula following Salvage Laryngectomy
}

\author{
Shinichi Ohba ${ }^{1 *}$, Mitsuhisa Fujimaki ${ }^{1}$, Masataka Kojima ${ }^{1}$, Junkichi Yokoyama ${ }^{2}$, Katsuhisa \\ Ikeda $^{1}$ \\ ${ }^{I}$ Department of Otorhinolaryngology-Head and Neck Surgery, Juntendo University Faculty of Medicine, Tokyo, \\ Japan \\ ${ }^{2}$ Otorhinolaryngology-Head and Neck surgery, Edogawa Hospital
}

*Corresponding Author: Shinichi Ohba M.D., Ph D, Department of Otorhinolaryngology-Head and Neck Surgery, Juntendo University Faculty of Medicine, 2-1-1 Hongo, Bunkyo-ku, Tokyo 113-8421, Japan, E-mail: sooba@juntendo.ac.jp

\begin{abstract}
Background: Pharyngocutaneous fistula $(P C F)$ is the most common and severe complication after salvage total laryngectomy. This study describestheusefulness of a pectoralis major muscle flap (PMMF) in reducing PCF developing rates after salvage total laryngectomy.
\end{abstract}

Methods: We describe an onlay PMMF technique. This technique includes neopharynx, which is primarily closed andcovered with PMMF.

Result: All four patients successfully underwent salvage total laryngectomy using the onlay PMMF technique. PCF was not observed in any of the four patients. The use of PMMF is considered to significantly decrease the rate of PCF formation.

Conclusion: The onlay PMMF method for salvage total laryngectomy was a feasible and easy technique thatmight prevent PCF formation.

Keywords: Pectoralis Major Muscle Flap (PMMF), Salvage Total Laryngectomy (STL), Pharyngocutaneous Fistula $(P C F)$.

\section{INTRODUCTION}

Pharyngocutaneous fistula (PCF) is a common and severe complication after salvage total laryngectomy. The rate of pharyngocutaneous fistula is about $65 \%$ when chemoradiation therapy is performed before surgery [1][2][3][4]. Such a complication results in prolonged time to oral feeding, hospitalization, and additional surgery that decreases the quality of life (QOL).

To prevent such a complication, minimal tissue dissection, avoiding neck dissection or relying on supers elective neck dissection when necessary, and using a muscle flap like the strap muscle or a sternocleidmastoid muscle flap used for repairing the defect have been performed in our department. However, the rate of pharyngocutaneous fistula was $61.1 \%$ after salvage total laryngectomy in our department.

There have been some reports about reducing the risk of PCF formation after STL
$[3][4][5][6][7][8]$. One technique is the useof a pectoralis major muscle flap (PMMF) onlay method [3][4][7]. The pectoralis major musculocutaneous flap (PMMCF) is feasible for the reconstruction of head and neck defects. This flap is easy to harvest, reliable and has stable circulation of the blood. The efficacy of a pectoralis major muscle flap (PMMF) for salvage total laryngectomy has been described previously in some papers [3][4][7][9][10][11]. A detailed description of the surgical procedure and the advantage of this method are presented.

\section{MATERIAL AND METHODS}

Four patients (4 males), ranging from 58 to 70years of age, underwent salvage total laryngectomy over a one year period. The clinical characteristics of the four cases are shown in Table1. The prophylactic use of PMMF for total laryngectomy was employed. The surgical technique is described as follows. Total laryngectomy is done with primary 
closure of the neopharynx. The blood supply of the cut end of the mucosa should be confirmed when completing the total laryngectomy. When there is insufficient mucosa remaining for primary closure $(3 \mathrm{~cm}$ in width; the thickness of a little finger in the pharyngeal cavity), apectoralis myocutaneous flap (PMMCF) or other myocutaneous flap (e.g. submental island flap, infrahyoid myocutaneous flap) should be applied. The PMMF is harvested immediately by an anterior thoracic incision. The subcutaneous tissue and pectoralis major muscle are divided widely. After identification of the lower border of the pectoralis major muscle, the plane between the pectoralis major muscle and ribs or the pectoralis minor muscle is dissected. The undersurface of the pectoralis major muscle is lifted up to expose the thoracoacromial vessels through the fascia. Under direct vision of the vessels, enough muscle flap to fully cover the neopharynx is elevated. PMMF is moved to the neck through a subcutaneous tunnel and stitched to the rest of pharyngeal constrictor muscle and supra-hyoid muscles. To prevent pedicle strangulation, the sternal head of the sternocleidomastoid muscle should be cut. Though the muscle placed in the neck is bulky, primary skin closure is safely done with permanent tracheostomal plasty.

We assessed the patients' characteristics, operation time, related complications, time to initiation of oral feeding and length of hospital stay.

\section{Results}

The outcome and data of the postoperative period are shown in Table1. No flap loss was documented. Among four patients, no patient developed pharyngocutaneous fistula compared to eleven out of eighteen patients $(61.1 \%)$ in the control group of total laryngectomy with primary closure.

Table1. Patient Characteristics

\begin{tabular}{cccccccc}
\hline age & sex & primary & surgery & $\begin{array}{c}\text { Surgical time } \\
\text { (flap elevation }\end{array}$ & complication & $\begin{array}{c}\text { oral feeding } \\
\text { time in days }\end{array}$ & $\begin{array}{c}\text { Hospital } \\
\text { stay }\end{array}$ \\
\hline 70 & M & Larynx & TL & $3: 28(0: 39)$ & none & 13 & 23 \\
62 & M & hypopharynx & $\begin{array}{c}\text { TL } \\
\text { Rt.ND }\end{array}$ & $5: 40(0: 33)$ & none & 10 & 17 \\
60 & M & larynx & $\begin{array}{c}\text { TL } \\
\text { Tt.ND }\end{array}$ & $4: 57(0: 44)$ & none & 15 & 25 \\
58 & M & larynx & TL & $3: 58(0: 39)$ & none & 13 & 19 \\
\hline
\end{tabular}

(Table2)The surgical time of laryngectomy with PMMF was not significantly longer than that for standard total laryngectomy (Table3). The average time for harvesting and transposing the flap to the neck was 38.75 minutes. The mean time to successful initiation of oral feeding was 12.75 days. The mean length of total hospital stay was 21.0 days. On the other hand, oral feeding time and length of hospital stay in patients with PCF was 95.5 days and 76.2 days, respectively.

Table2. Comparison of pharyngocutaneous fistula (PCF) formation between the group with the PMMF onlay method and the group with primary closure. No PCF formation was observed in the PMMF group $(p=0.027<0.05 ;$ Pearson's chi-square test $)$

\begin{tabular}{lccc}
\hline & PCF(+) & PCF(-) & \\
\hline PMMF onlay & 0 & 4 & 4 \\
$\begin{array}{l}\text { Primary } \\
\text { closure }\end{array}$ & 11 & 7 & 18 \\
\hline
\end{tabular}

Table3. Comparison of surgical time between the group with the PMMF onlay method and the control group(primary closure). Addition of PMMF elevation and transposition to salvage total laryngectomy(STL) or STL with neck dissection did not affect the total surgical time ( $t$ test).

\begin{tabular}{lcccc}
\hline & $\begin{array}{c}\text { PMMF onlay } \\
(\text { min })\end{array}$ & $\begin{array}{c}\text { Primary closure } \\
(\text { min })\end{array}$ & \\
\hline TL & $233.9^{2} \pm 91.7$ & $223 \pm 21.2$ & NS \\
& & & & \\
TL + ND & $318.5 \pm 30.4$ & $308.6 \pm 73.7$ & NS \\
\hline
\end{tabular}

\section{DISCUSSION}

Recently chemoradiation therapy has become common for locally advanced laryngeal and hypopharyngeal cancer for the purpose of organ preservation. Accordingly, salvage surgery including salvage total laryngectomy (STL) for treatment failure has also increased. However, The incidence of postoperative pharyngocutaneous fistula (PCF) after STL is a significant problem carrying the risk of postoperative infection, tissue necrosis and blowout of a major vessel including carotid artery[12]. Furthermore, such complications result in decreased quality of life, prolonged hospital stay and greater care expenditure.

To reduce the risk of pharyngocutaneous fistula after salvage total laryngectomy, several techniques have been reported [3][4][5][6][7][811][13]. The basic concept is that non-irradiated 
tissue is transferred to the surgical field to reinforce the fragile lesion like a pharyngeal suture and to repair the defect.

Several studies on the prophylactic use of a pectoralis major muscle flap (PMMF) for STL have been described. Most of them showed a significant reduction of PCF by using PMMF [3-11]. However, if wider resection of the hypopharynx is needed because of tumor extension, a pectoral is major myocutaneous flap (PMMCF) should be applied to repair the pharyngeal defect. Guimarães A.V. et al reported that the vascularization of the pectoralis muscle may be so robust and reliable that a PMMF is capable of sealing off the pharyngotomy [10]. Furthermore, the deep fascia surrounding the pectoralis muscle is rich in hyaluronan, which may be beneficial during the earliest stages of wound healing [10].

In the present study, harvesting the PMMF did not affect the total operation time compared to STL with only primary closure. PMMF or PMMCF may be used for head and neck cancer patients with severe co morbidity, when the reliability of a flap and a shorter surgical time are required [14]. In our department, PMMF or PMMCF can be harvested within30-60 min. There was no wound complication in the donor site in this series. When PMMF is applied, the donor site can be closed easily, unlike PMMCF which may require a split skin graft. Therefore, the PMMF onlay technique was considered to be a minimally invasive method for STL.

Some disadvantages of the PMMF onlay technique have been reported, including reduced neck mobility, reduced swallowing function or impaired post laryngectomy speech because of the muscle bulk.

In addition, the excessive bulk of the muscle may be cosmetically less acceptable. If PCF develops following STL with PMMF onlay method, PMMCF of the contra lateral side or other flaps should be considered to close the fistula. This might be a disadvantage in that the flap choices used as a backup are reduced. Even if such disadvantages occur, patients may accept the situation compared to the possible development of PCF requiring prolonged hospitalization.

On the other hand, there havebeen reports describing that PMMF does not contribute to reducing the PCF rate [3][15]. However, Patel, et. al stated that even if a fistula occurs after STL when using the PMMF onlay method, it tends to close much faster than cases with primary closure[16].

Nonetheless, the rate of pharyngocutaneous fistula (PCF) formation rate is certainly high in salvage total laryngectomy. Therefore, some kind of countermeasure to prevent PCF formation is needed. The prophylactic use of a PMMF appears to be an easy, simple and feasible method to reduce the risk of PCF after salvage total laryngectomy.

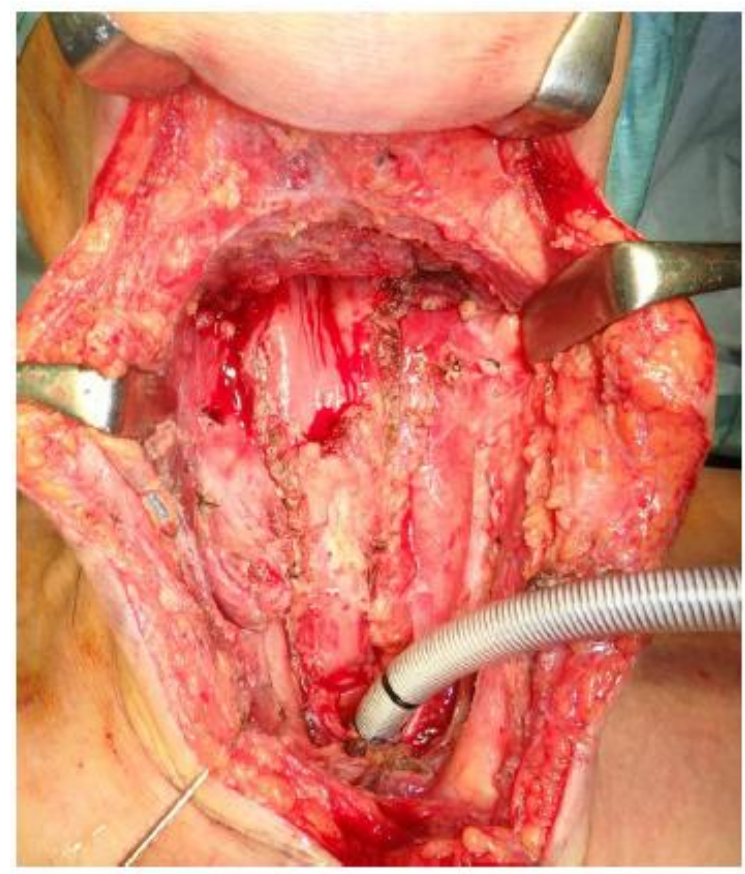

Figure1. After removal of the larynx. At least a $3 \mathrm{~cm}$ transverse diameter of the residual pharyngeal mucosa is needed for the primary closure.

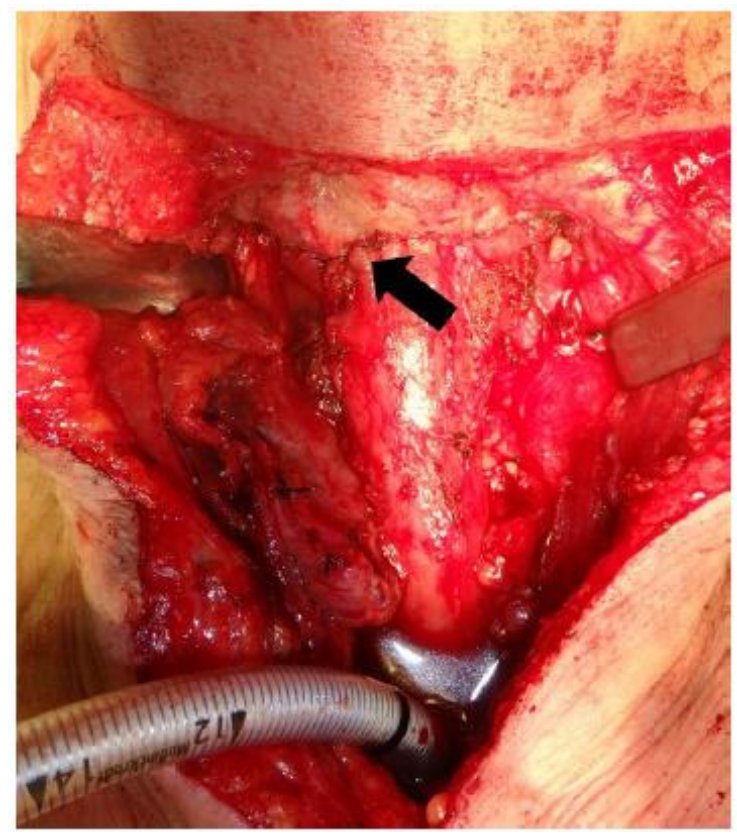

Figure2. A T-shaped closure was done. A horizontal/transverse closure is preferred if there is not undue tension on the suture line 


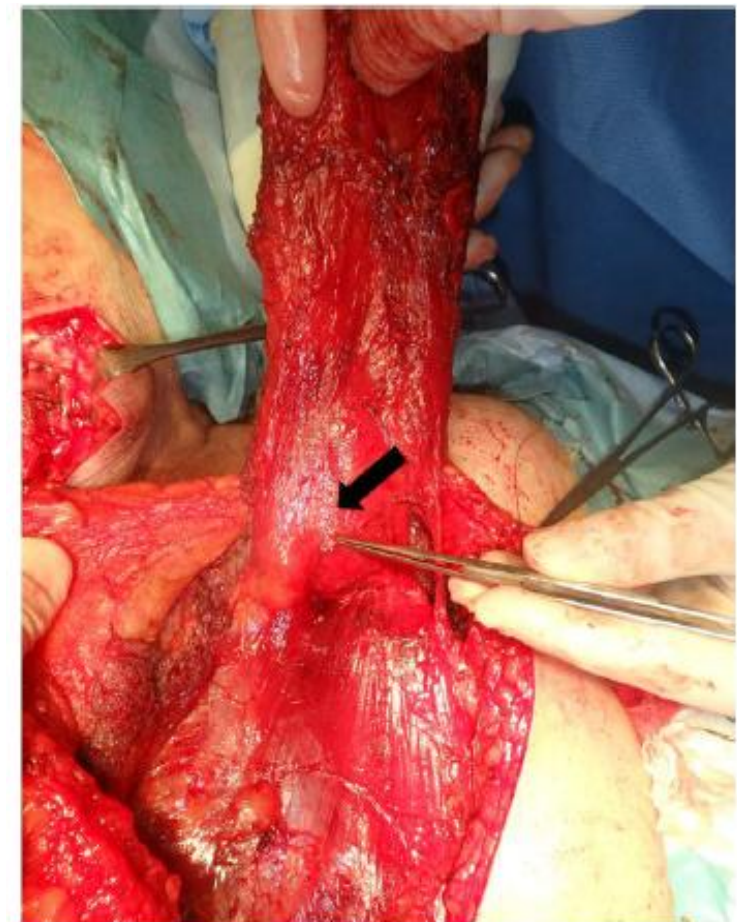

Figure3. Elevation of the pectoralis major muscle flap (PMMF). Vascular pedicle (pectoral branch of the thoracoacromial artery) is confirmed on the deep surface of the pectoralis major muscle.

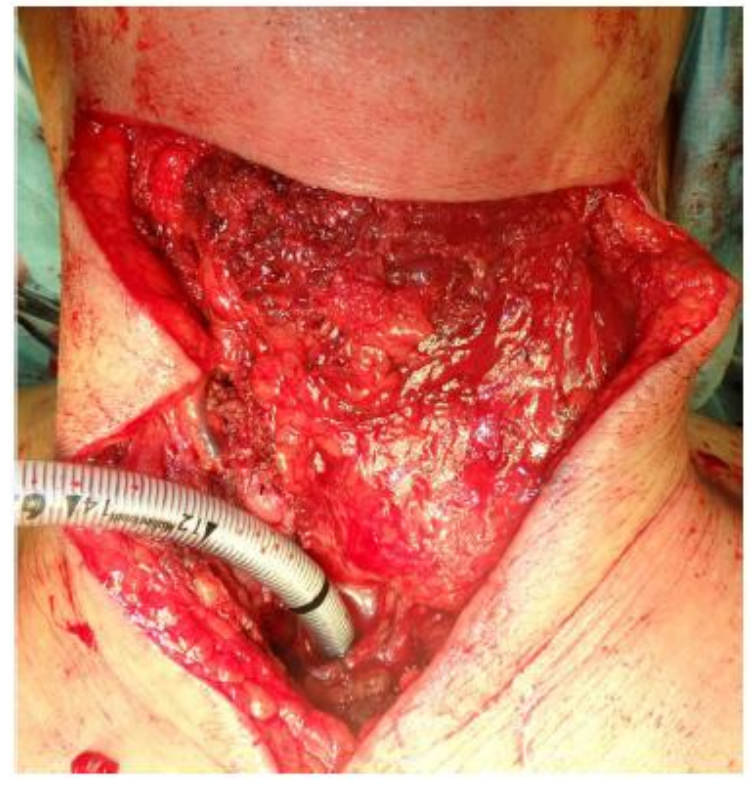

Figure4. PMMF was transferred over the clavicle through a subcutaneous tunnel and fully covered the suture line of the pharyngeal mucosa. PMMF was fixed with some stiches

\section{CONCLUSION}

The prophylactic use of a PMMF is an easy, simple and feasible method for salvage total laryngectomy. A little ingenuity with thirty minutes or so in STL may help not only in maintaining the QOL of patients but in decreasing the need for additional procedures and treatment.

\section{AUTHORS' CONTRIBUTIONS}

SO conceived of the study. SO prepared and edited the manuscript. MF and MK contributed to the acquisition of data. MF and MK performed the statistical analysis. MK and KI conducted the interpretation of data. SO and JY revised the final version of the manuscript. All authors read and approved the final manuscript.

\section{REFERENCES}

[1] Süslü N, Senirli RT, Günaydın RÖ, Özer S, Karakaya J, Hoşal AŞ. Pharyngocutaneous fistula after salvage laryngectomy.Acta Otolaryngol. 2015 Jun;135(6):615-21.

[2] Paydarfar JA, Birkmeyer NJ (2006) Complications in head and neck surgery: a meta-analysis of postlaryngectomy pharyngocutaneous fistula. Arch Otolaryngol Head Neck Surg 132:67-72.

[3] Cömert E, Tunçel Ü, Torun MT, Kiliç C, Cengiz AB, Sencan Z, Kaya M. Pectoralis major myofascial flap in salvage laryngectomy.J Laryngol Otol. 2014 Aug;128(8):714-9.

[4] Gendreau-Lefèvre AK1, Audet N1, Maltais S1, Thuot F1. Prophylactic pectoralis major muscle flap in prevention of pharyngocutaneous fistula in total laryngectomy after radiotherapy.Head Neck. 2015 Sep; 37(9):1233-8.

[5] Sundaram K, Wasserman J (2009) Prevention of unplanned pharyngocutaneous fistula in salvage laryngectomy. Otolaryngol Head Neck Surg 141:645-647 16.

[6] Calli C, Pinar E, Oncel S (2011) Pharyngocutaneous fistula after total laryngectomy: less common with mechanical stapler closure. Ann Otol Rhinol Laryngol 120:339-344.

[7] Lukas Anschu "tz1 ,Lluı 's Nisa,Olgun Elicin, Beat Bojaxhiu,Marco Caversaccio,Roland Giger. Pectoralis major myofascial interposition flap prevents postoperative pharyngocutaneous fistula in salvage total laryngectomy. Eur Arch Otorhinolaryngol (2016) 273:3943-3949.

[8] Sakai A, Okami K, Sugimoto R, Ebisumoto K, Yamamoto H, Maki D, Atsumi T, Iida M. Prevention of wound complications in salvage pharyngolaryngectomy by the use of wellvascularized flaps. Acta Otolaryngol. 2012 Jul;132(7):778-82.

[9] Gendreau-Lefèvre AK, Audet N, Maltais S, Thuot F. Prophylactic pectoralis major muscle flap in prevention of pharyngocutaneous fistula in total laryngectomy after radiotherapy.Head Neck. 2015 Sep;37(9):1233-8.

[10] Guimarães AV, Aires FT, Dedivitis RA, Kulcsar MA, Ramos DM, Cernea CR, Brandão LG. Efficacy of pectoralis major muscle flap for pharyngocutaneous fistula prevention in 
salvage total laryngectomy: A systematic review.Head Neck. 2016 Apr;38 Suppl 1:E2317-21.

[11] Gilbert MR1, Sturm JJ, Gooding WE, Johnson JT, Kim S. Pectoralis major myofascial onlay and myocutaneous flaps and pharyngocutaneous fistula in salvage laryngectomy. Laryngoscope. 2014 Dec;124(12):2680-6.

[12] Eugene N. Myers, MD The Management of Pharyngocutaneous Fistula. Arch Otolaryngol. 1972;95(1):10-17.

[13] Dedivitis RA, Aires FT, Pfuetzenreiter EG Jr, Castro MA, Guimarães AV. Stapler suture of the pharynx after total laryngectomy.Acta Otorhinolaryngol Ital. 2014 Apr;34(2):94-8.

[14] Ribeiro Salles Vanni CM1, de Matos LL, Faro Junior MP, Ledo Kanda J, Cernea CR, Garcia
Brandão L, Pinto FR. Enhanced morbidity of pectoralis major myocutaneous flap used for salvage after previously failed oncological treatment and unsuccessful reconstructive head and neck surgery. ScientificWorldJournal. Epub 2012 May 3.

[15] Gil Z, Gupta A, Kummer B, Cordeiro PG, Kraus DH, Shah JP, Patel SG The role of pectoralis major muscle flap in salvage total laryngectomy. Arch Otolaryngol Head Neck Surg. 2009 Oct;135(10):1019-23.

[16] Patel UA, Moore BA, Wax M, Rosenthal E, Sweeny L, Militsakh ON, Califano JA, Lin AC, Hasney CP, Butcher RB, Flohr J, Arnaoutakis D, Huddle M, Richmon JD. Impact of pharyngeal closure technique on fistula after salvage laryngectomy.JAMA Otolaryngol Head Neck Surg. 2013 Nov;139(11):1156-62.

Citation: Shinichi Ohba, Mitsuhisa Fujimaki, Masataka Kojima, Junkichi Yokoyama \& Katsuhisa Ikeda. A Novel Method of Preventing Pharyngocutaneous Fistula following Salvage Laryngectomy. ARC Journal of Surgery.2018; 4(1):15-19. doi: dx.doi.org/10.20431/2455-572X. 0401004.

Copyright: (c) 2018 Authors. This is an open-access article distributed under the terms of the Creative Commons Attribution License, which permits unrestricted use, distribution, and reproduction in any medium, provided the original author and source are credited. 